\title{
Two Classes of Hot Jupiter Atmospheres
}

\author{
Jonathan J. Fortney \\ Dept. of Astronomy \& Astrophysics, UCO/Lick Observatory \\ University of California, Santa Cruz, CA, USA \\ email: jfortney@ucolick.org
}

\begin{abstract}
We highlight the potential importance of gaseous $\mathrm{TiO}$ and $\mathrm{VO}$ opacity on the highly irradiated close-in giant planets. The day-side atmospheres of these planets may naturally fall into two classes that are somewhat analogous to the M- and L-type dwarfs. Those that are warm enough to have appreciable opacity due to $\mathrm{TiO}$ and VO gases we term the "pM Class" planets, and those that are cooler, such that $\mathrm{Ti}$ and $\mathrm{V}$ are predominantly in solid condensates, we term "pL Class" planets. The optical spectra of pL Class planets are dominated by neutral atomic $\mathrm{Na}$ and $\mathrm{K}$ absorption. We discuss a connection between temperature inversions and large day/night temperature contrasts for the pM Class planets. Around a Sun-like primary, for solar composition, this boundary likely occurs at $\sim 0.04-0.05 \mathrm{AU}$, but we discuss important uncertainties. The difference in the observed day/night contrast between $v$ And $\mathrm{b}$ (pM Class) and HD 189733b (pL Class) is naturally explained in this scenario.
\end{abstract}

\section{Introduction}

The blanket term "hot Jupiter" or even the additional term "very hot Jupiter" belies the diversity of these highly irradiated planets. Each planet likely has its own unique atmosphere, interior structure, and accretion history. The relative amounts of refractory and volatile compounds in a planet will reflect the parent star abundances, nebula temperature, total disk mass, location of the planet's formation within the disk, duration of its formation, and its subsequent migration (if any). During these early stages of exoplanet characterization, it is worthwhile to try and understand patterns that are emerging from the data returned by Spitzer and Hubble. In Fortney et al. (2008) we analyzed secondary eclipse and light curve data for hot Jupiter planets in an attempt to understand the diverse physics and chemistry of these atmospheres. Here we briefly summarize this work, as well as investigate a few additional areas including detection of optical flux. Those interested should also read the prescient paper by Hubeny et al. (2003) on TiO/VO in hot Jupiters, as well as Burrows et al. (2008), which covers some of the same ground as Fortney et al. (2008).

\section{Background, Terminology, and Observations}

Since irradiation is perhaps the most important factor in determining the atmospheric properties of these planets, we examine the insolation levels of the known Jupiter-class transiting planets. Figure 1 illustrates the stellar flux incident upon the planets as a function of planet mass. In this plot diamonds indicate transiting planets and triangles indicate other interesting hot Jupiters, for which Spitzer Space Telescope data exist, but which do not transit. The first known transiting planet, HD 209458b, is seen to be fairly representative in terms of incident flux.

Fortney et al. (2008) argue that based on the examination of a few physical processes, two classes of hot Jupiter atmospheres emerge. Equilibrium chemistry abundances, 


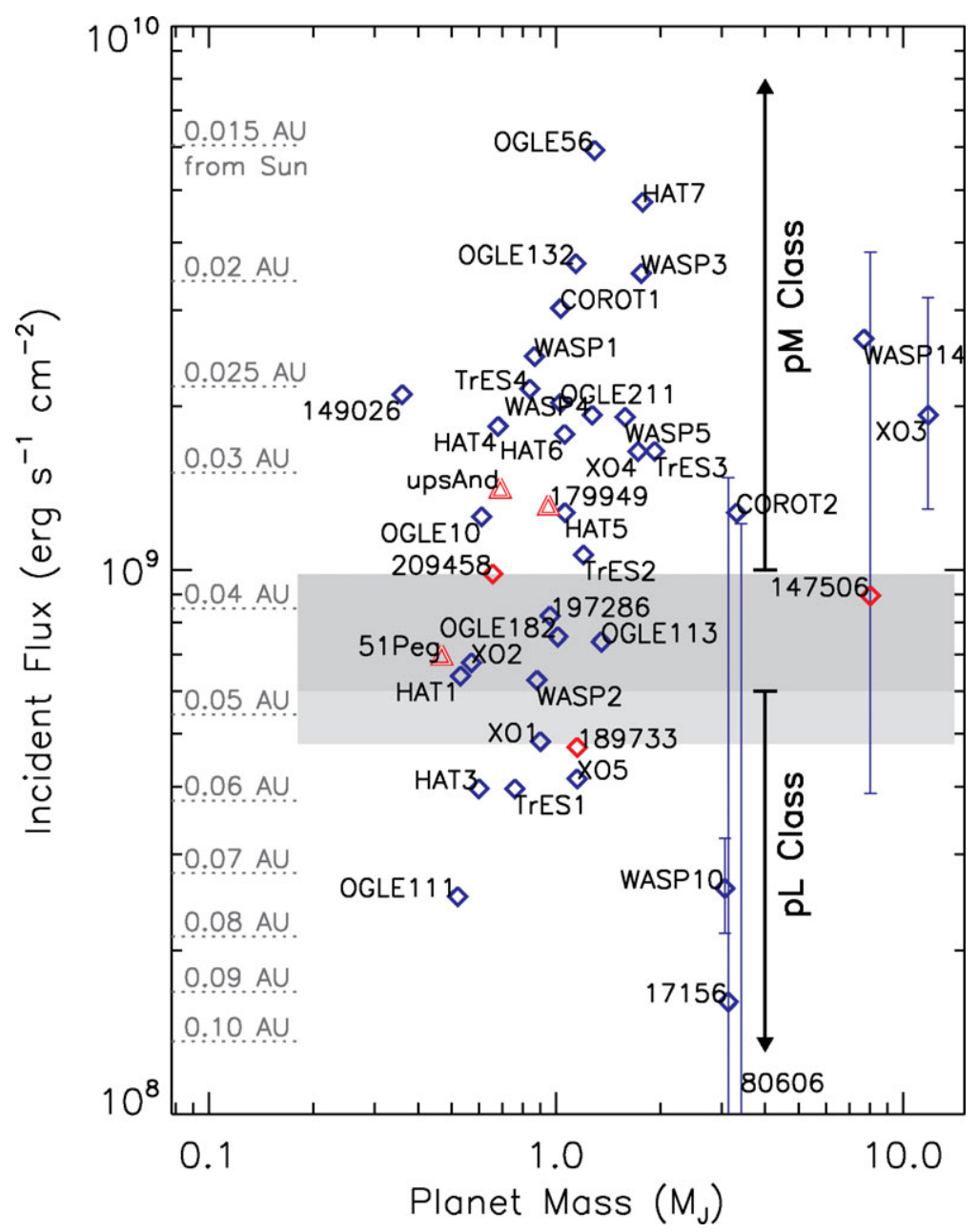

Figure 1. Flux incident upon a collection of hot Jupiter planets, as of 30 June 2008. The labeled dotted lines indicate the distance from the Sun that a planet would have to be to intercept this same flux. Diamonds indicate the transiting planets while triangles indicate non-transiting systems (with minimum masses plotted). The error bars for some planets indicate the variation in flux that each receives over their eccentric orbits. Flux levels for pM Class and pL Class planets are shown, with the dark shaded region around $\sim 0.04-0.05 \mathrm{AU}$ indicating a possible transition region (based more on data available in Fall 2007, than on theory), from Fortney et al. (2008). The lighter shaded region reaches down to XO-1b.

the depth to which incident flux will penetrate into a planet's atmosphere, and the radiative time constant as a function of pressure and temperature in the atmosphere all naturally define two classes these irradiated planets. Those planets that are warmer than required for condensation of titanium $(\mathrm{Ti})$ and vanadium $(\mathrm{V})$-bearing compounds will possess a temperature inversion at low pressure due to absorption of incident flux by TiO and VO (a hot stratosphere, as first found by Hubeny et al. 2003), and will appear "anomalously" bright in secondary eclipse at mid-infrared wavelengths. Furthermore, these planets will have large day/night effective temperature contrasts and their optical flux will be dominated by thermal emission. We will term these very hot Jupiters the "pM 
Class," $†$ meaning gaseous $\mathrm{TiO}$ and VO are the prominent absorbers of optical flux, similar to $\mathrm{M}$ dwarfs. Planets with temperatures below the condensation temperature curve of $\mathrm{Ti}$ and $\mathrm{V}$ bearing compounds will have relatively smaller secondary eclipse depths in the mid infrared, significantly smaller day/night effective temperature contrasts, and atmospheric dynamics will lead to a complex redistribution of absorbed energy. We term these hot Jupiter planets the "pL Class," since once TiO/VO are removed, alkalis become the prominent optical absorbers (Burrows, Marley, \& Sharp 2000), similar to L dwarfs. Published Spitzer data are fairly consistent with this picture, as we describe below. Planets that are thought to have inversions, based on comparisons of infrared data (predominantly from Spitzer) to spectral models include:

- HD 209458b (Knutson et al. 2008a; Burrows et al. 2007)

- HD 149026b (Harrington et al. 2007; Fortney et al. 2006b)

- XO-1b (Machalek et al. 2008)

- TrES-4b (H. Knutson, et al., in prep.)

- HD 179949 (Barnes et al. 2008)

Tentative analyses presented at this meeting indicated inversions for 2 other planets at incident fluxes similar to HD 209458b. Planets that do not have inversions include:

- HD 189733b (Charbonneau et al. 2008; Barman 2008)

- TrES-1 (Charbonneau et al. 2005; Fortney et al. 2005; Burrows et al. 2005, 2008)

The observed inversion for XO-1b is particularly interesting, as its irradiation level is quite similar to that of HD 189733b. Why does one have an inversion and the other does not? We are not yet able to answer this definitely. We note that the $1 \sigma$ error bars on the stellar $T_{\text {eff }} \mathrm{s}$ and radii could actually lead to a $10 \%$ difference in incident flux between them. Also, planetary atmospheric metallicity could play a role, especially for the temperatures for condensation of $\mathrm{Ti}$ and $\mathrm{V}$. At the modest temperatures of XO-1b, $\mathrm{TiO} / \mathrm{VO}$ can probably only be found at the substellar point (where it is hottest), if the molecules make it above a possible cold trap (Hubeny et al. 2003). Perhaps only VO is present, rather than $\mathrm{TiO}$, since $\mathrm{V}$ condenses more gradually with reduced temperature (Lodders 2002). Also, these molecules may not be the exclusive strong optical absorbers. Observations of cool planets HAT-P-3b, XO-5b, WASP-10b, as well as those intermediate in flux between HD 209458b and XO-1b, will be very important.

We note that the relatively homogenized atmospheric temperatures of HD 189733b (Knutson et al. 2007), contrasted with the large day/night contrasts of HD 179949b (Cowan et al. 2007) and $v$ And b (Harrington et al. 2006) seem to reinforce the connection between temperature inversions and larger day/night contrasts. Additional light curve data will be important. Also, the Sing et al. (2008) transmission spectrum of HD 209458b appears to show a small amount of $\mathrm{TiO} / \mathrm{VO}$ on the cool limb of the planet, indicating that these molecules may be causing its temperature inversion.

\section{Model Predictions}

A key difference between the atmospheres of the pL Class planets and pM Class planets is the pressure at which the absorption and emission of flux occurs (Fortney et al. 2008). This can be shown by plotting the pressure that corresponds to a calculated brightness temperature. This is shown as a function of wavelength in Figure 2. In general thermal emission arises from a characteristic pressure level roughly an order of magnitude greater in a pL Class atmosphere than in a pM Class atmosphere.

$\dagger$ We note that some TV Sci-Fi fans have used "M Class" for decades to describe life-bearing terrestrial planets. See http://www.memory-alpha.org/en/wiki/Class_M for details. 


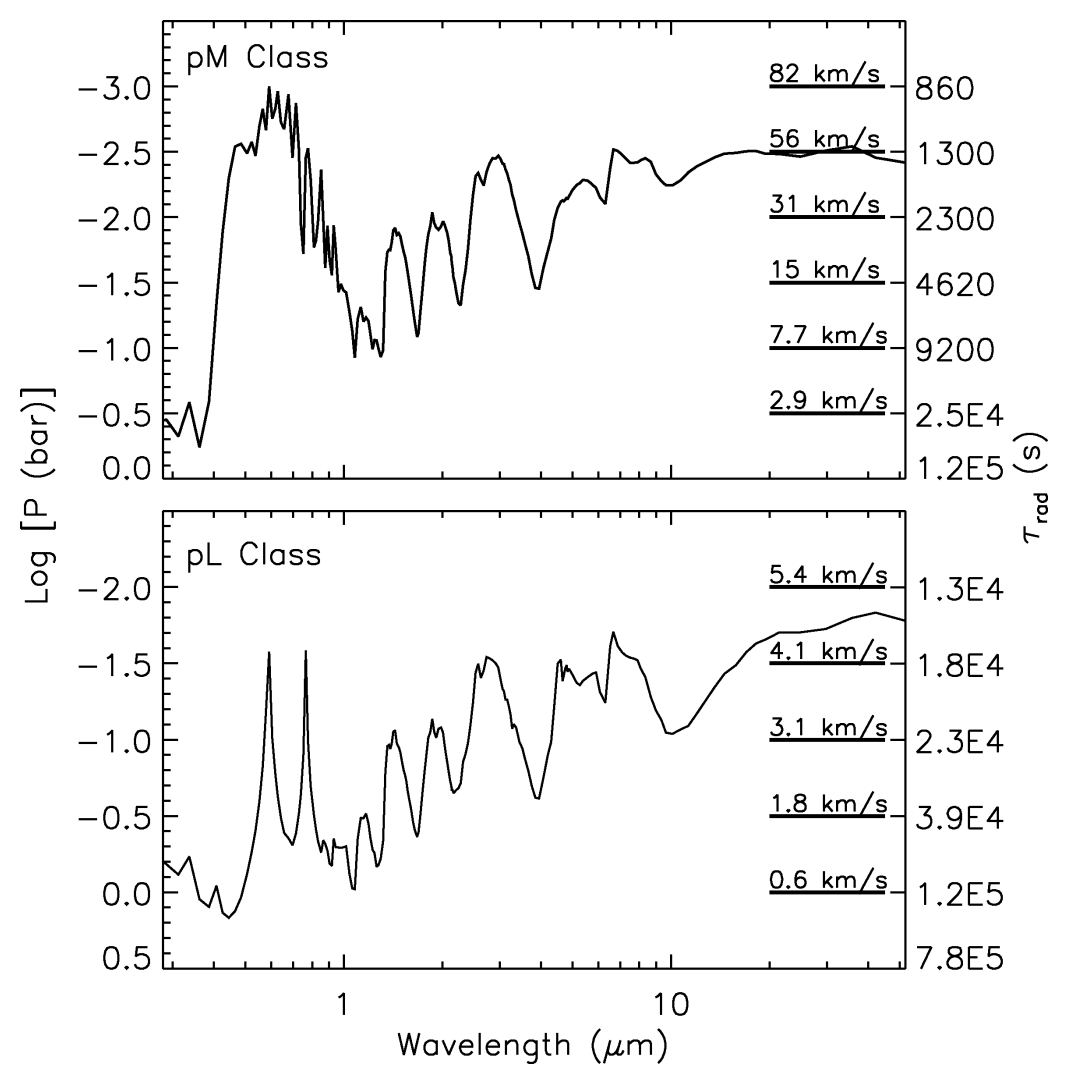

Figure 2. Both figures show, as a function of wavelength, the approximate pressure at which $\tau=2 / 3$. Note the differences in the y-axes. Top: Planet at $0.03 \mathrm{AU}$ which has an inversion induced by absorption by $\mathrm{TiO} / \mathrm{VO}$. Bottom: Planet at $0.05 \mathrm{AU}$ that lacks an inversion. The right ordinate (which is not linear) shows the corresponding radiative time constant at each major tick mark from the pressure axis. The labeled gray lines indicate the wind speed that would be necessary to give an advective time scale equal to the given radiative constant.

What effect this may have on the dynamical redistribution of energy can be considered after calculation of the radiative time constant, $\tau_{\text {rad }}$. In the Newtonian cooling approximation a temperature disturbance relaxes exponentially toward radiative equilibrium with a characteristic time constant (see Showman \& Guillot 2002; Fortney et al. 2008).

The right y-axis in Figure 2 is the $\tau_{\text {rad }}$ appropriate for a given pressure in the atmosphere. While those for the pM Class range from only $10^{3}-10^{4} \mathrm{~s}$, those for the pL Class range from $10^{4}-10^{5}$ seconds. This $\sim 10 \times$ difference in timescale is a consequence of both the hotter temperatures and lower pressures of the pM Class photospheres.

One can also define an advective time scale, a characteristic time for moving atmospheric gas a given planetary distance. A common definition is $\tau_{\text {advec }}=\frac{R_{\mathrm{p}}}{U}$, where $U$ is the wind speed and $R_{\mathrm{p}}$ is the planet radius (Showman \& Guillot 2002; Seager et al. 2005). If one sets $\tau_{\text {advec }}=\tau_{\text {rad }}$ and sets $R_{\mathrm{p}}=1 R_{\mathrm{J}}$, one can derive the wind speed $U$ that would be necessary to advect atmospheric gas before a $\tau_{\text {rad }}$ has elapsed. This is also shown in Figure 2. As previously discussed (Seager et al. 2005; Fortney et al. 2006a; Barman 2008) since the efficiency of energy redistribution and the depth to which one "sees" are wavelength dependent, the shape and times of maxima and minima in thermal emission light curves will be a function of wavelength. Examination of Figure 2 shows that for a pM Class planet winds speeds of dozens of $\mathrm{km} \mathrm{s}^{-1}$ would be necessary for 


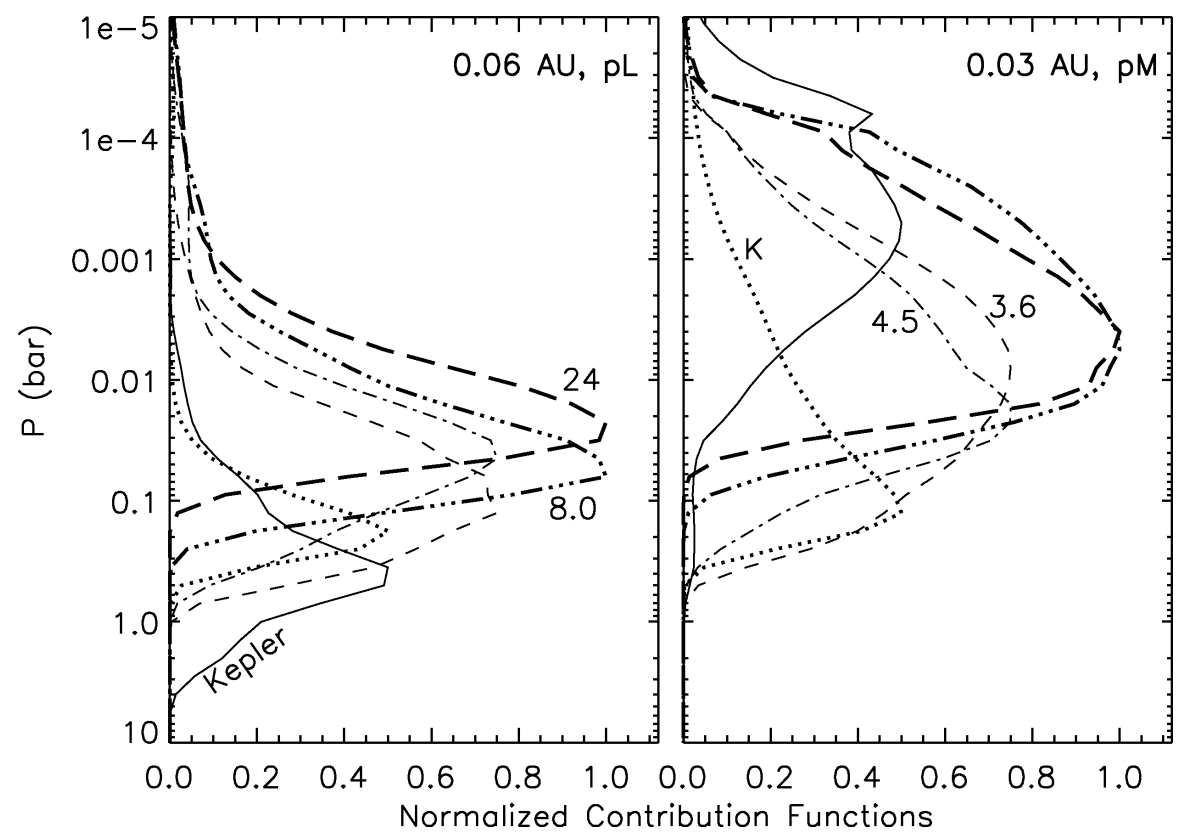

Figure 3. Contribution functions of a planet at $0.03 \mathrm{AU}$ and $0.06 \mathrm{AU}$ from the Sun. The same line scheme in used in both panels. Kepler, K, and Spitzer bands are labeled. This provides more detailed information of emission as a function of pressure, in particular bands, than Figure 2.

advection to dominate over cooling/heating! For a pL Class planet more modest winds speeds of several $\mathrm{km} \mathrm{s}^{-1}$ are needed. Several $\mathrm{km} \mathrm{s}^{-1}$ winds are in line with predictions of a number of 2D and 3D dynamical models for hot Jupiter atmospheres (see Showman et al. 2007, for a review). These calculated wind speeds are far below those needed to advect air before it radiatively heats/cools in a pM Class atmosphere.

We can take another look at the pressures from which day-side emission originates in these atmospheres. Figure 3 shows normalized contribution functions $(c f)$ for the thermal flux from the atmosphere (e.g. Chamberlain \& Hunten 1987; Griffith et al. 1998):

$$
c f(P)=B(\lambda, T)=\frac{\mathrm{d} e^{-\tau}}{\mathrm{d} \log (P)}
$$

The band-averaged $c f$ s show the fractional contribution of various pressures to the outgoing thermal radiation in the chosen bands. Note that the peak in the $c f$ differs from the pressure one would estimate from simply assigning the brightness temperature to a given pressure (Chamberlain \& Hunten 1987). The $c f$ s show that there is considerable overlap in the contributions of the flux between the various Spitzer bands. The enhanced opacity of the day-side of a pM Class atmosphere (due to $\mathrm{TiO} / \mathrm{VO}, \mathrm{H}_{2} \mathrm{O}$, and $\mathrm{H}_{2} \mathrm{CIA}$ ) leads to emission from lower pressures in these atmospheres.

\section{Expectations for Optical Flux}

Even the most sensitive searches for optical light from hot Jupiters have yielded only upper limits (see Rowe et al. 2007, and Figure 4, for HD 209458b). While scattered ("reflected") light from these atmospheres may be small (Fortney et al. 2008; Burrows et al. 2008), for the hot Jupiters thermal emission at optical wavelengths can be appreciable (Marley et al. 1999; López-Morales \& Seager 2007). In Figure 5 we plot optical 


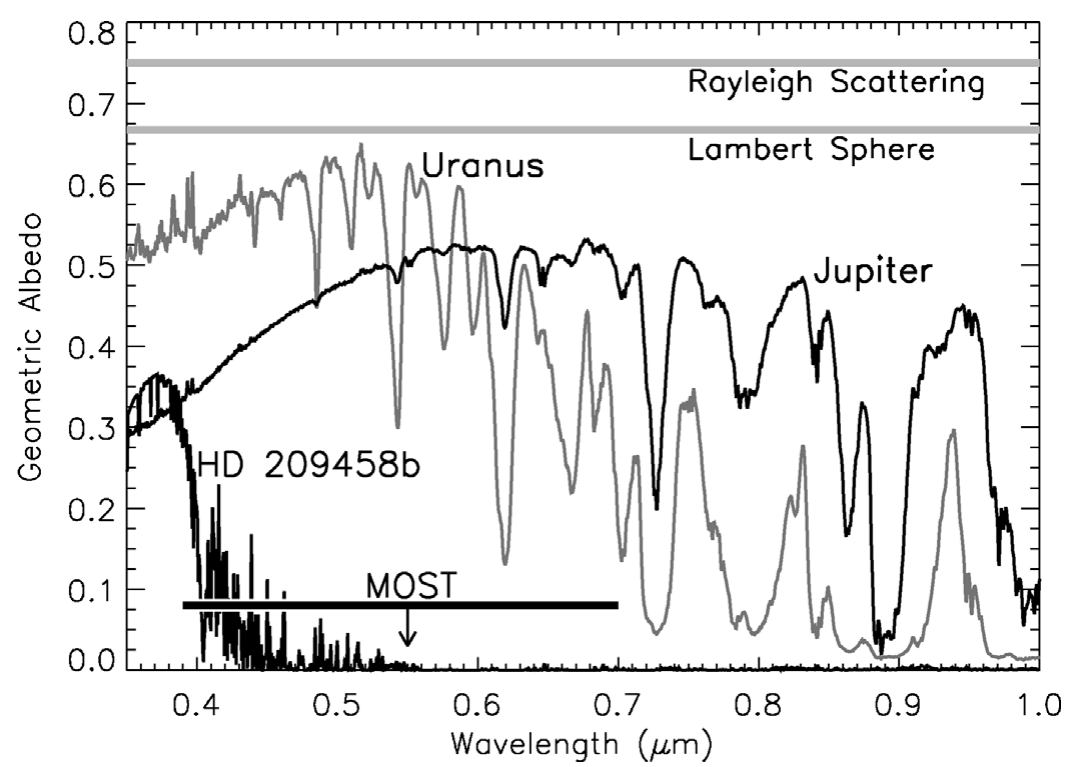

Figure 4. Observed geometric albedo of Uranus and Jupiter (Karkoschka 1994), compared to one possible model of HD 209458b which ignores the thermal emission contribution to the observed geometric albedo, and the $1 \sigma$ upper limit from MOST (Rowe et al. 2007). The geometric albedo of a deep Rayleigh scattering atmosphere and a Lambertian sphere are shown as well.

planet-to-star flux ratios for models with orbital separations of 0.0125 to $0.06 \mathrm{AU}$ from the Sun. The total (scattered+thermal) planet flux is shown with thick lines, while the thermal component only is in thin lines. Planet-to-star flux ratios approach 0.001 in the red for planets even more irradiated than OGLE-TR-56b (the flux equivalent of a planet at $0.015 \mathrm{AU}$ from the Sun). For the hottest planets the gaseous opacity at optical wavelengths is dominated by bands of $\mathrm{TiO}$ and VO. These models have temperature inversions, so the bands here are seen in emission. For the cooler planet at $0.05 \mathrm{AU}$, nearly all $\mathrm{TiO} / \mathrm{VO}$ is condensed and an inversion does not occur in this simple $1 \mathrm{D}$ day-side model. The optical spectrum is dominated by absorption by $\mathrm{Na}$ and $\mathrm{K}$, with very modest absorption by extremely depleted $\mathrm{TiO} / \mathrm{VO}$ seen. By $0.06 \mathrm{AU}, \mathrm{TiO} / \mathrm{VO}$ is absent.

\section{Conclusions}

The data so far points towards an incredible diversity of atmospheres within the hot Jupiter planets. Future characterization, across the widest possible range in incident bolometric fluxes, UV fluxes, and orbital period should shed much additional light on these exotic atmospheres. We advocate a robust program with Warm Spitzer to determine if planets possess temperature inversions (from short-wavelength IRAC colors) as well as long integrations to measure day/night temperature contrasts. Optical and near-IR transmission spectra will continue to yield important insights into atmospheric chemistry and abundances.

\section{References}

Barman, T. S. 2008, ApJ, 676, L61

Barnes, J. R., Barman, T. S., et al. 2008, A\&A in press, astro-ph/0806.0298

Burrows, A., Budaj, J., \& Hubeny, I. 2008, ApJ, 678, 1436 


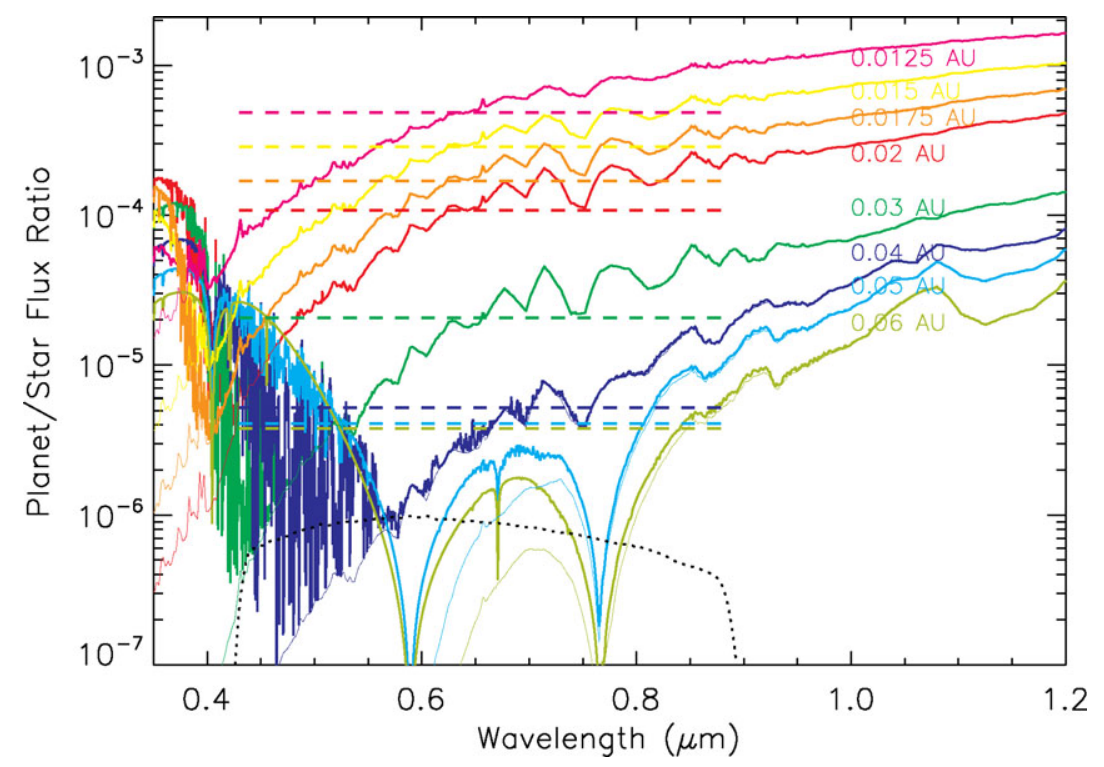

Figure 5. Model planet-to-star flux ratios at secondary eclipse for close-in planets from 0.06 to 0.0125 AU. Planets have a surface gravity of $15 \mathrm{~cm} \mathrm{~s}^{-2}$, radius $1.2 R_{\mathrm{J}}$. The thick solid curves are the combined scattered and thermal planet flux. Thin solid lines are the thermal component only. Thick dashed lines show the band-average flux ratio in the Kepler band. Thermal emission dominates scattered flux at small orbital distances.

Burrows, A., Hubeny, I., Budaj, J., Knutson, H. A., \& Charbonneau, D. 2007, ApJ, 668, L171 Burrows, A., Hubeny, I., \& Sudarsky, D. 2005, ApJ, 625, L135

Burrows, A., Marley, M. S., \& Sharp, C. M. 2000, ApJ, 531, 438

Chamberlain, J. W. \& Hunten, D. M. 1987, Orlando, Academic Press Inc Int'l Geophysics Series Charbonneau, D., et al. 2005, ApJ, 626, 523

Charbonneau, D., et al. 2008, ApJ submitted, astro-ph/0802.0845, 802

Cowan, N. B., Agol, E., \& Charbonneau, D. 2007, MNRAS, 379, 641

Fortney, J. J., Cooper, C. S., Showman, A. P., et al. 2006a, ApJ, 652, 746

Fortney, J. J., Lodders, K., Marley, M. S., \& Freedman, R. S. 2008, ApJ, 678, 1419

Fortney, J. J., Marley, M. S., Lodders, K., Saumon, D., \& Freedman, R. 2005, ApJ, 627, L69

Fortney, J. J., Saumon, D., Marley, M. S., et al. 2006b, ApJ, 642, 495

Griffith, C. A., Yelle, R. V., \& Marley, M. S. 1998, Science, 282, 2063

Harrington, J., Hansen, B. M., et al. 2006, Science, 314, 623

Harrington, J., Luszcz, S., Seager, S., Deming, D., \& Richardson, L. J. 2007, Nature, 447, 691

Hubeny, I., Burrows, A., \& Sudarsky, D. 2003, ApJ, 594, 1011

Karkoschka, E. 1994, Icarus, 111, 174

Knutson, H. A., Charbonneau, D., et al. 2008a, ApJ, 673, 526

Knutson, H. A., Charbonneau, D., et al. 2007, Nature, 447, 183

Lodders, K. 2002, ApJ, 577, 974

López-Morales, M. \& Seager, S. 2007, ApJ, 667, L191

Machalek, P., McCullough, et al. 2008, ApJ in press, astro-ph/0805.2418

Marley, M. S., Gelino, C., Stephens, D., Lunine, J. I., \& Freedman, R. 1999, ApJ, 513, 879

Rowe, J. F., Matthews, J. M., et al. 2007, ApJ in press, astro-ph/0711.4111

Seager, S., et al. 2005, ApJ, 632, 1122

Showman, A. P. \& Guillot, T. 2002, A\&A, 385, 166

Showman, A. P., et al. 2007, Extreme Solar Systems, in press, astro-ph/0710.2930

Sing, D. K., et al. 2008, ApJ in press, astro-ph/0802.3864 